\title{
The ALMA-PILS Survey: Formaldehyde deuteration in warm gas on small scales toward IRAS 16293-2422 B
}

\author{
M. V. Persson ${ }^{1,2}$, J. K. Jørgensen ${ }^{3}$, H. S. P. Müller ${ }^{4}$, A. Coutens ${ }^{5}$, E. F. van Dishoeck ${ }^{2,6}$, V. Taquet ${ }^{7}$, H. Calcutt ${ }^{2}$, \\ M. H. D. van der Wiel ${ }^{8}$, T. L. Bourke ${ }^{9}$, and S. F. Wampfler ${ }^{10}$ \\ ${ }^{1}$ Department of Space, Earth and Environment, Chalmers University of Technology, Onsala Space Observatory, 43992 Onsala, \\ Sweden \\ e-mail: magnpe@chalmers.se \\ 2 Leiden Observatory, Leiden University, PO Box 9513, 2300 RA Leiden, The Netherlands \\ 3 Centre for Star and Planet Formation, Niels Bohr Institute \& Natural History Museum of Denmark, University of Copenhagen, \\ Øster Voldgade 5-7, 1350 Copenhagen K, Denmark \\ ${ }^{4}$ I. Physikalisches Institut, Universität zu Köln, Zülpicher Str. 77, 50937 Köln, Germany \\ 5 Laboratoire d'Astrophysique de Bordeaux, Univ. Bordeaux, CNRS, B18N, allée Geoffroy Saint-Hilaire, 33615 Pessac, France \\ 6 Max-Planck Institute für extraterrestrische Physik (MPE), Giessenbachstrasse, 85748 Garching, Germany \\ 7 INAF-Osservatorio Astrofisico di Arcetri, Largo E. Fermi 5, 50125 Firenze, Italy \\ 8 ASTRON Netherlands Institute for Radio Astronomy, PO Box 2, 7990 AA Dwingeloo, The Netherlands \\ 9 SKA Organization, Jodrell Bank Observatory, Lower Withington, Macclesfield, Cheshire SK11 9DL, UK \\ ${ }^{10}$ Center for Space and Habitability (CSH), University of Bern, Sidlerstrasse 5, 3012 Bern, Switzerland
}

Received 31 July 2017 / Accepted 8 November 2017

\begin{abstract}
Context. The enhanced degrees of deuterium fractionation observed in envelopes around protostars demonstrate the importance of chemistry at low temperatures, relevant in pre- and protostellar cores. Formaldehyde is an important species in the formation of methanol and more complex molecules.

Aims. Here, we aim to present the first study of formaldehyde deuteration on small scales around the prototypical low-mass protostar IRAS 16293-2422 using high spatial and spectral resolution Atacama Large Millimeter/submillimeter Array (ALMA) observations. We determine the excitation temperature, abundances and fractionation level of several formaldehyde isotopologues, including its deuterated forms.

Methods. Excitation temperature and column densities of formaldehyde in the gas close to one of the components of the binary were constrained through modeling of optically thin lines assuming local thermodynamical equilibrium. The abundance ratios were compared to results from previous single dish observations, astrochemical models and local ISM values.

Results. Numerous isotopologues of formaldehyde are detected, among them $\mathrm{H}_{2} \mathrm{C}^{17} \mathrm{O}$, and $\mathrm{D}_{2}^{13} \mathrm{CO}$ for the first time in the ISM. The large range of upper energy levels covered by the $\mathrm{HDCO}$ lines help constrain the excitation temperature to $106 \pm 13 \mathrm{~K}$. Using the derived column densities, formaldehyde shows a deuterium fractionation of $\mathrm{HDCO} / \mathrm{H}_{2} \mathrm{CO}=6.5 \pm 1 \%, \mathrm{D}_{2} \mathrm{CO} / \mathrm{HDCO}=12.8_{-4.1}^{+3.3} \%$, and $\mathrm{D}_{2} \mathrm{CO} / \mathrm{H}_{2} \mathrm{CO}=0.6(4) \pm 0.1 \%$. The isotopic ratios derived are ${ }^{16} \mathrm{O} /{ }^{18} \mathrm{O}=805_{-79}^{+43},{ }^{18} \mathrm{O} /{ }^{17} \mathrm{O}=3.22_{-0.3}^{+0.2}$, and ${ }^{12} \mathrm{C} /{ }^{13} \mathrm{C}=56_{-11}^{+8}$.

Conclusions. The $\mathrm{HDCO} / \mathrm{H}_{2} \mathrm{CO}$ ratio is lower than that found in previous studies, highlighting the uncertainties involved in interpreting single dish observations of the inner warm regions. The $\mathrm{D}_{2} \mathrm{CO} / \mathrm{HDCO}$ ratio is only slightly larger than the $\mathrm{HDCO} / \mathrm{H}_{2} \mathrm{CO}$ ratio. This is consistent with formaldehyde forming in the ice as soon as $\mathrm{CO}$ has frozen onto the grains, with most of the deuteration happening toward the end of the prestellar core phase. A comparison with available time-dependent chemical models indicates that the source is in the early Class 0 stage.
\end{abstract}

Key words. astrochemistry - stars: formation - stars: protostars - ISM: molecules - ISM: individual objects: IRAS 16293-2422

\section{Introduction}

Among molecular abundance ratios, the deuterium fractionation (D/H ratio) is commonly used to infer the formation conditions (e.g., Roberts \& Millar 2000). In general, a high D/H ratio indicates a low temperature, and a low $\mathrm{D} / \mathrm{H}$ ratio indicates a high temperature during formation. The deuterium-to-hydrogen ratio in the local interstellar medium (ISM) is $2.0 \pm 0.1 \times 10^{-5}$ (Prodanović et al. 2010). Many molecules are formed on the surfaces of dust grains, and once it is cold enough for $\mathrm{CO}$ to freeze out onto the grains, the deuterium chemistry is enhanced. This means that any molecules formed after $\mathrm{CO}$ freezeout are expected to have high levels of deuterium fractionation (e.g., Taquet et al. 2014). Single dish observations of various molecules toward deeply embedded protostars have shown high deuterium fractions, above 10\% (e.g., van Dishoeck et al. 1995; Parise et al. 2006). Optical depth and source size effects (beam dilution) are significant for single dish observations of lines of the main isotopologue which makes the uncertainty in the fractions large. High sensitivity and high resolution interferometric observations have the possibility to circumvent these problems since they probe smaller scales, close to the forming star where the temperature is high enough to sublimate the grain surface ices completely (i.e., $T \gtrsim 100 \mathrm{~K}$ ). The higher sensitivity makes it possible to detect weaker lines of minor isotopologues where optical depth effects are less important. 
Observations of water have revealed a low deuterium fractionation (e.g., $\mathrm{HDO} / \mathrm{H}_{2} \mathrm{O}$ ratio) on small scales (warm gas) in young protostellar envelopes. While the cold gas shows a $\mathrm{HDO} / \mathrm{H}_{2} \mathrm{O}$ ratio of a few $\%$, the warm gas has a ratio closer to $0.1 \%$ (e.g., Persson et al. 2014; Coutens et al. 2012). Furthermore, the water deuterium fractionation of the warm gas in the inner region toward the protostar NGC 1333 IRAS 2A shows a $\mathrm{D}_{2} \mathrm{O} / \mathrm{HDO}$ ratio significantly higher than the $\mathrm{HDO} / \mathrm{H}_{2} \mathrm{O}$ ratio (seven times higher, Coutens et al. 2014). Furuya et al. (2016) explain the observed ratios by modeling the pre-stellar core physical and chemical evolution. The gas-phase deuteration processes are inefficient early on due to the higher $\mathrm{H}_{2} \mathrm{o} / \mathrm{p}$-ratio raising the destruction rate of the main seed molecule for deuteration processes, $\mathrm{H}_{2} \mathrm{D}^{+}$(Pagani et al. 1992). However, the deuteration processes through $\mathrm{H}_{2} \mathrm{D}^{+}$increase as soon as the o/p-ratio decreases and other molecules that destroy it freezes out (e.g. CO, Furuya et al. 2015). This and the low temperature results in higher gas-phase atomic $\mathrm{D} / \mathrm{H}$ ratio which in turn causes more $\mathrm{D}$ atoms to accrete (mainly through $\mathrm{H}_{2} \mathrm{D}^{+}$) on the grains (first pointed out by Tielens 1983). This drives the hydrogen-surface deuterium chemistry, however the total production rate of water is significantly decreased at this point, thus for water the deuteration is low. This explains both the low absolute deuteration that water has, and the relative ratios between the deuterated forms. Whether other molecules show similar trends is not yet clear. However many molecules are thought to form later, once $\mathrm{CO}$ has frozen onto the grains and could thus show different ratios in this model.

Formaldehyde is an important molecule, not only for constraining physical conditions in the gas of star-forming regions, but also as an intermediary in the formation path to complex organic molecules. $\mathrm{H}_{2} \mathrm{CO}$ formation in ices was shown experimentally and models highlighted that it proceeds at a significantly higher rate in ices than in gas-phase reactions (Roberts et al. 2004). Thus it is thought that formaldehyde is mainly formed on the surface of dust grains once $\mathrm{CO}$ has frozen onto the grains through the hydrogen addition reactions $\mathrm{CO}+\mathrm{H} \rightarrow$ $\mathrm{HCO}$ and then $\mathrm{HCO}+\mathrm{H} \rightarrow \mathrm{H}_{2} \mathrm{CO}$ seen in the laboratory (Watanabe \& Kouchi 2002; Fuchs et al. 2009). The deuterated forms of $\mathrm{H}_{2} \mathrm{CO}$ can be obtained through subsequent substitution reactions involving D-atoms, that is, $\mathrm{H}_{2} \mathrm{CO}+\mathrm{D} \rightarrow \mathrm{HDCO}+\mathrm{H}$ and $\mathrm{HDCO}+\mathrm{D} \rightarrow \mathrm{D}_{2} \mathrm{CO}+\mathrm{H}$. They can also be obtained through abstraction and addition reactions through the $\mathrm{HCO}$ and DCO radical (e.g., Tielens 1983), which has been shown to be efficient at low temperature in ices (Hidaka et al. 2009). Naturally, just as with $\mathrm{H}_{2} \mathrm{CO}$ there is also a direct channel from $\mathrm{CO}$ through DCO, that is, the tunneling reaction $\mathrm{CO}+\mathrm{D} \rightarrow \mathrm{DCO}$, with subsequent addition reactions to form $\mathrm{D}_{2} \mathrm{CO}$ and $\mathrm{HDCO}$.

Assuming a grain surface formation route, Turner (1990) and later Charnley et al. (1997) estimated the relation between abundance ratios of the deuterated isotopologues of formaldehyde, assuming equal transmission probabilities for the $\mathrm{CO}+\mathrm{H}$ and $\mathrm{CO}+\mathrm{D}$ reaction channels and neglecting abstraction reactions, to be

$$
\frac{\mathrm{D}_{2} \mathrm{CO}}{\mathrm{HDCO}}=\frac{1}{4} \frac{\mathrm{HDCO}}{\mathrm{H}_{2} \mathrm{CO}} \text {. }
$$

While this was enough to explain the observed deuterium fractionation of formaldehyde at the time, it seems now that the situation is slightly more complex for deeply-embedded protostars, and that substitution and abstraction (with subsequent addition) reactions are important.

In this paper we study the deuterium fractionation of formaldehyde $\left(\mathrm{H}_{2} \mathrm{CO}\right)$ on small solar-system scales toward one of the sources in the Class 0 multiple system IRAS 16293-2422, located in the $\rho$ Ophiuchus star forming region at a distance of 120 pc (Loinard et al. 2008). The deuterium fractionation is determined through line analysis of various deuterated and nondeuterated isotopologues of formaldehyde. IRAS 16293-2422 is a protostellar binary with a separation of about $5^{\prime \prime}$ (600 AU), where the SE source is referred to as "A" and the NW source as "B". Several studies have shown IRAS 16293-2422 to be a chemically rich source, with a wealth of complex organic molecular species (e.g., Bottinelli et al. 2004; Kuan et al. 2004; Bisschop et al. 2008; Jørgensen et al. 2011) and with thermal water (Persson et al. 2013) associated with both sources. Recent detections include the prebiotic molecule glycolaldehyde (Jørgensen et al. 2012), its deuteration (Jørgensen et al. 2016), and the detection of ethylene oxide, acetone, propanal, methyl isocyanate, and formamide (Coutens et al. 2016; Lykke et al. 2017; Martín-Doménech et al. 2017; Ligterink et al. 2017). For an extended overview of the literature on IRAS 16293-2422 and a review of the ALMA-PILS survey see Jørgensen et al. (2016).

Measurements of the deuterium fractionation of formaldehyde toward IRAS 16293-2422 have previously been limited to single dish observations. Using the JCMT and the CSO van Dishoeck et al. (1995) derived a $\mathrm{HDCO} / \mathrm{H}_{2} \mathrm{CO}$ ratio of $14 \pm 7 \%$ in a $20^{\prime \prime}$ beam (2400 AU), taking nonLTE effects into account. Assuming the same excitation temperature for both $\mathrm{HDCO}$ and $\mathrm{H}_{2} \mathrm{CO}$ an even higher ratio of $\mathrm{HDCO} / \mathrm{H}_{2} \mathrm{CO}=33_{-18}^{+42} \%$ was obtained with the same observations by Parise et al. (2006). The $\mathrm{D}_{2} \mathrm{CO} / \mathrm{H}_{2} \mathrm{CO}$ ratio was mapped around the source and constrained to $3 \%$ toward the edge of the envelope and peaking at $16 \%$ toward a position roughly one single dish beam south (Ceccarelli et al. 1998, 2001). Further single dish observations, also with signatures of self-absorption, constrain the ratios to $\mathrm{HDCO} / \mathrm{H}_{2} \mathrm{CO}=13-16 \%, \mathrm{D}_{2} \mathrm{CO} / \mathrm{H}_{2} \mathrm{CO}=5-$ $6 \%$, and $\mathrm{D}_{2} \mathrm{CO} / \mathrm{HDCO}=33-40 \%$ (Loinard et al. 2000). However, for these studies opacity effects and multiple contributions to the emission within the beam might affect the column densities by factors of between two and a few (Parise et al. 2006), thus the abundances in cold gas need to be more accurately constrained for a proper comparison with ratios derived here for the warm gas on small scales.

Formaldehyde was mapped at high angular resolution toward IRAS 16293-2422 by Schöier et al. (2004), showing some of the uncertainties involved in deriving abundances of formaldehyde with single dish telescopes, and highlighting the importance of higher resolution. The formation and deuteration of formaldehyde, methanol and other species were studied through chemical modeling by Taquet et al. (2012) and more recently by Taquet et al. (2014). The more recent models trace the deuteration with time from the prestellar core stage to the end of the deeply-embedded stage. The models cannot reproduce the high levels of formaldehyde deuteration previously observed. To further test the chemical models and assess the importance of grain surface formation as well as the various formation paths it is important to accurately constrain the relative abundances of the different deuterated forms of formaldehyde. Interferometric observations of the inner warm region have the possibility to constrain the abundances where the grain surface ice has just been completely sublimated, without the pitfalls of single dish observations.

This paper is laid out as follows. In Sect. 2 details of the observations and spectroscopic data are given together with a description of the analysis method. Section 3 gives the result of the analysis; the measured excitation temperature and the column densities of the various isotopologues of formaldehyde. 
In Sect. 4 the derived abundances and isotopologue ratios are discussed with previous measurements and chemical models in mind. This is followed by a short summary and outlook on future prospects in Sect. 5 .

\section{Observations and analysis}

This study is based on observations from the Protostellar Interferometric Line Survey (PILS ${ }^{1}$ ), an ALMA Band 7 unbiased spectral line survey with complete coverage between 329.15 and $362.90 \mathrm{GHz}$ at $0.244 \mathrm{MHz}$ resolution. For details, including observing conditions, calibration and imaging, see Jørgensen et al. (2016). The observations include both the main array of $12 \mathrm{~m}$ dishes, and the $7 \mathrm{~m}$ dishes of the Atacama Compact Array (ACA). The observations cover both sources in the IRAS 16293-2422 multiple system. The PILS spectrum toward Source A shows lines with larger line widths $\left(2-8 \mathrm{~km} \mathrm{~s}^{-1}\right.$ FWHM) than for Source B (around $1 \mathrm{~km} \mathrm{~s}^{-1}$ FWHM) making line identification much easier toward the B source, also when comparing to the Galactic Center and high-mass protostars previously observed in the same fashion. The inclusion of the ACA observations fills in the shorter baselines and thus the extended emission encapsulating the sources with a maximum recoverable scale of $\sim 13^{\prime \prime}$. The final spectral image cubes have a rms of 4-8 $\mathrm{mJy} \mathrm{beam}^{-1} \mathrm{~km} \mathrm{~s}^{-1}$, a synthesized beam with a FWHM of $0 \prime$.5 and a flux calibration uncertainty of $\sim 5 \%$. The phase center is located half-way between the large scale binary A and B $\left(\alpha_{\mathrm{J} 2000}=16^{\mathrm{h}} 32^{\mathrm{m}} 22^{\prime \prime} \cdot 72 ; \delta_{\mathrm{J} 2000}=-24^{\circ} 28^{\prime} 34^{\prime \prime} .3\right)$ and the field of view covers roughly $15^{\prime \prime}$.

The observed spectra toward the two main sources show a large number of lines. The unprecedented sensitivity and richness of the observed spectrum have so far facilitated several new detections and constraints on isotopologue ratios. So far the abundances and excitation conditions for acetone, propanal and methyl isocyanate (Lykke et al. 2017; Ligterink et al. 2017), and also the deuterium fraction of formamide, glycolaldehyde, ketene and other oxygen-bearing (complex) organic molecules have been presented (Coutens et al. 2016; Jørgensen et al. 2016, 2017).

Similar to the analysis presented in Lykke et al. (2017), Coutens et al. (2016), and Ligterink et al. (2017), the spectrum from an offset position toward Source B was extracted. Located at $\alpha_{\mathrm{J} 2000}=16^{\mathrm{h}} 32^{\mathrm{m}} 22^{\prime \prime} .58, \delta_{\mathrm{J} 2000}=-24^{\circ} 28^{\prime} 32^{\prime \prime} .8$ it is $\sim 0^{\prime \prime} .5$ offset (i.e., one beam) in the southwest direction relative to the continuum peak of source B (see Fig. 1). The main reason for this is that toward the continuum peak, lines are affected by varying degrees of absorption and continuum optical depth, complicating the line identification and analysis of the abundances (see contours in the right panel of Fig. 1).

\subsection{Laboratory spectroscopy data}

The transition frequencies and other data (including partition function values) of all forms of formaldehyde were taken from the CDMS database (Müller et al. 2001, 2005). The $\mathrm{H}_{2} \mathrm{CO}$, $\mathrm{H}_{2} \mathrm{C}^{17} \mathrm{O}$ and $\mathrm{H}_{2} \mathrm{C}^{18} \mathrm{O}$ entries are based on Müller \& Lewen (2017), and the $\mathrm{H}_{2}^{13} \mathrm{CO}$ entry on Müller et al. (2000c). Important additional data are from Brünen et al. (2003); Bocquet et al. (1996) in the case of $\mathrm{H}_{2} \mathrm{CO}$ along with ground state combination differences employed in Müller et al. (2000a). Müller et al. (2000b); Cornet \& Winnewisser (1980a) contributed additional data to the $\mathrm{H}_{2} \mathrm{C}^{18} \mathrm{O}$ entry, the latter also to those of $\mathrm{H}_{2} \mathrm{CO}$

\footnotetext{
1 http://youngstars.nbi.dk/PILS
}

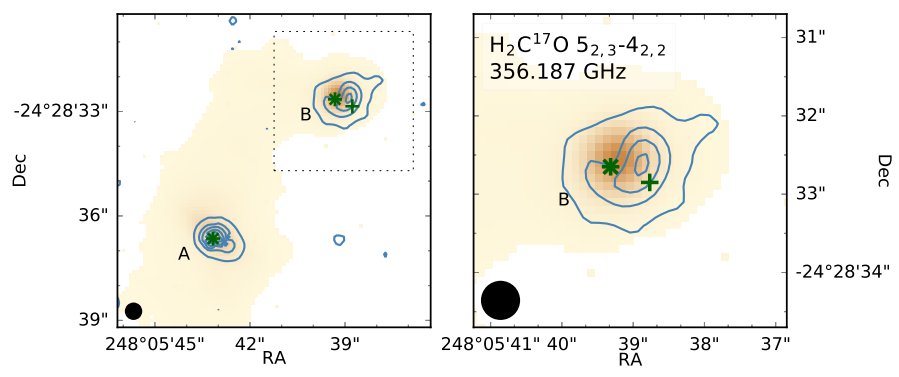

Fig. 1. PILS ALMA continuum map of the IRAS 16293-2422 system at $850 \mu \mathrm{m}$. The background image shows the continuum emission, the contours indicate the $\mathrm{H}_{2} \mathrm{C}^{17} \mathrm{O} 5_{2,3}-4_{2,2}$ line $\left(E_{\mathrm{u}}=98.5 \mathrm{~K}\right)$ integrated emission, the star is the position of the continuum peak, and the plus sign the position of the extracted spectrum (one beam, i.e., 0"5, away from the continuum). The continuum image is cut off at $15 \mathrm{mJy}(3 \sigma)$ and the line contours start at $25 \mathrm{mJy} \mathrm{km} \mathrm{s}^{-1}(5 \sigma)$ in steps of $120 \mathrm{mJy} \mathrm{km} \mathrm{s}{ }^{-1}$.

and $\mathrm{H}_{2}^{13} \mathrm{CO}$. The $\mathrm{H}_{2} \mathrm{C}^{17} \mathrm{O}$ entry employed previous data from Cornet et al. (1980b) and from Flygare \& Lowe (1965).

Entries of the deuterated isotopologues, $\mathrm{HDCO}, \mathrm{D}_{2} \mathrm{CO}$, $\mathrm{HD}^{13} \mathrm{CO}, \mathrm{D}_{2}^{13} \mathrm{CO}, \mathrm{HDC}^{18} \mathrm{O}$, and $\mathrm{D}_{2} \mathrm{C}^{18} \mathrm{O}$, were based on the very recent analyses by Zakharenko et al. (2015). The analyses took into account earlier data for five of these six isotopologues (no earlier data for $\mathrm{HDC}^{18} \mathrm{O}$ ) from Dangoisse et al. (1978) as well as extensive data for $\mathrm{HDCO}$ and $\mathrm{D}_{2} \mathrm{CO}$ from Bocquet et al. (1999). Far-infrared data of $\mathrm{D}_{2} \mathrm{CO}$ (Lohilahti \& Horneman 2004) and $D_{2}^{13} \mathrm{CO}$ (Lohilahti et al. 2005) were also employed for the CDMS entries.

\subsection{Abundances}

The abundances are estimated by computing a synthetic spectrum assuming local thermal equilibrium (LTE) and comparing it with the observed spectrum. In addition to this, CASSIS ${ }^{2}$ was used as a support tool, for example, to check for possible line blending from other species, which is the same method as in other PILS survey studies for example Coutens et al. (2016); Ligterink et al. (2017); Lykke et al. (2017). For HDCO a full grid in excitation temperature and column density is calculated and the coordinates of the minimum $\chi^{2}$ are taken as the best estimates. Because the excitation temperature is better constrained using HDCO than through the other isotopologues of formaldehyde, its resulting temperature is used to constrain the column density for all forms. This is done by varying the column density, calculating the integrated line flux for each line not affected by optical depth effects or significant blending by other species and comparing to the observed line flux by calculating the $\chi^{2}$ estimate. The uncertainty in abundance is estimated by finding the minimum $\chi^{2}$ for the lower and upper bound excitation temperature (i.e., 93 and 119 K, Fig. 2). The column densities are corrected for the continuum emission from the surrounding dense dust (by multiplying with a factor of 1.1658 , Jørgensen et al. 2016). A line width of $1.15 \mathrm{~km} \mathrm{~s}^{-1}$ (FWHM) and system velocity of $2.7 \mathrm{~km} \mathrm{~s}^{-1}$ were used (relevant for source B). The assumed line width agrees well with the observed line profiles for formaldehyde and is similar to that of other species (Coutens et al. 2016; Lykke et al. 2017).

The detected transitions of the various isotopologues, deuterated and non-deuterated are listed in Table A.1. The analysis focuses on $\mathrm{H}_{2} \mathrm{CO}$ (three lines detected) and several deuterated

\footnotetext{
2 Developed by IRAP-UPS/CNRS http://cassis.irap.omp.eu
} 


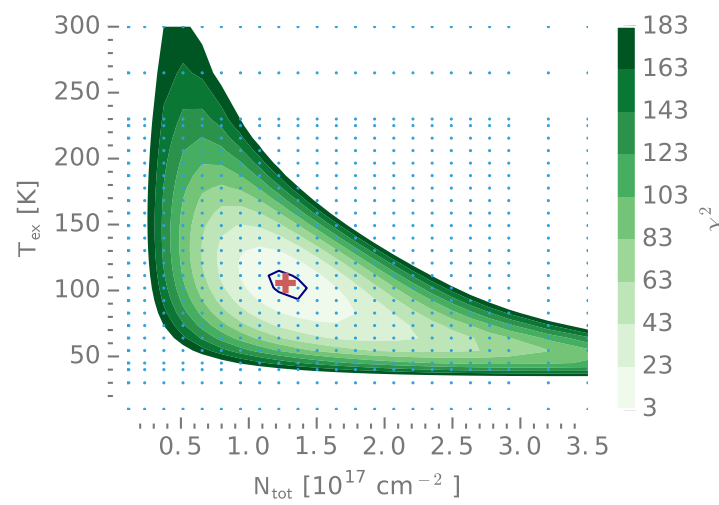

Fig. 2. Grid constraining the excitation temperature and column density of HDCO. The cross marks the best fit values, $1.3 \pm 0.2 \times 10^{17} \mathrm{~cm}^{-2}$ and $106 \pm 13 \mathrm{~K}$ from interpolation of the values within the marked area. The contour line marks the $\Delta \chi^{2}=2.3$ region. We note that the column density value has been corrected for the continuum. The dots show the evaluated column densities and excitation temperatures.

and non-deuterated isotopologues (number of detected lines in parenthesis): $\mathrm{H}_{2}{ }^{13} \mathrm{CO}(9), \mathrm{H}_{2} \mathrm{C}^{18} \mathrm{O}(9), \mathrm{H}_{2} \mathrm{C}^{17} \mathrm{O}(8), \mathrm{HDCO}(6)$, $\mathrm{HDC}^{18} \mathrm{O}$ (1 tentative), $\mathrm{D}_{2} \mathrm{CO}(11)$, and $\mathrm{D}_{2}{ }^{13} \mathrm{CO}(10)$.

\section{Results}

In the following sections we present the results of a detailed analysis of the column densities, the various isotopic ratios and the constraints on the excitation temperature. Only optically thin lines are included in the analysis to minimize any opacity effects.

\subsection{Excitation temperature}

The excitation temperature was determined to be $106 \pm 13 \mathrm{~K}$ by fitting the spectrum of HDCO, and the resulting temperature is used for all forms of formaldehyde to constrain their column density. The $\chi^{2}$ in a grid around the best fitting column density and excitation temperature is shown in Fig. 2. The region with an added $\Delta \chi^{2}=2.3$ is taken as the $1 \sigma$ error in $T_{\text {ex }}$ and $N_{\text {tot }}$ for HDCO (Avni 1976; Press et al. 2002). This temperature, together with the constrained column densities reproduce the lines for all isotopologues well. The figures in Appendix B show the LTE model for the best fitting column densities and the observed spectrum for all lines of all species, including non-detections.

In some studies of other sources a second temperature component is needed to reproduce the spectra of lines of both lower and higher upper energy levels (e.g., Isokoski et al. 2013). If a second temperature component were relevant for this study, for example, the low $E_{\mathrm{u}}$ lines would be systematically under or overproduced in the synthetic spectrum. Since this is not the case it is likely that only one temperature component is present. The other isotopologues were not as suitable to use for constraining the excitation temperature, the resulting parameter space of HDCO shows a clearer minimum than any of the other isotopologues investigated (Fig. 2). The spread in $E_{\mathrm{u}}$ of the detected and optically thin lines of HDCO makes it better at constraining the temperature. Using selected lines of $\mathrm{H}_{2} \mathrm{C}^{18} \mathrm{O}$ a less constrained excitation temperature is obtained $(90-200 \mathrm{~K})$. For the other isotopologues, it was possible to constrain the column density to high accuracy with the given excitation temperature.

In addition to the detected, optically thin HDCO lines (Table A.1) we also used upper limits of one non-detected HDCO transition at $348.965 \mathrm{GHz}\left(E_{\mathrm{u}}=1017.78 \mathrm{~K}, \log _{10}\left(A_{i j}\right)=\right.$ -4.5321 ) to constrain the excitation temperature (see Table A.2). Since the LTE model should not overproduce this line, it
Table 1. Best-fit continuum corrected column densities at $T_{\mathrm{ex}}=106 \pm$ $13 \mathrm{~K}$ for all the isotopologues of formaldehyde.

\begin{tabular}{|c|c|}
\hline Species & $N_{\text {tot }}\left(\mathrm{cm}^{-2}\right)$ \\
\hline $\mathrm{H}_{2} \mathrm{CO}$ & $1.9_{-0.2}^{+0.1} \times 10^{18}$ \\
\hline $\mathrm{H}_{2}{ }^{13} \mathrm{CO}$ & $3.6_{-1.1}^{+0.2} \times 10^{16}$ \\
\hline $\mathrm{H}_{2} \mathrm{C}^{17} \mathrm{O}$ & $7.2_{-0.6}^{+0.5} \times 10^{14}$ \\
\hline $\mathrm{H}_{2} \mathrm{C}^{18} \mathrm{O}$ & $2.3 \pm 0.1 \times 10^{15}$ \\
\hline $\mathrm{HDCO}$ & $1.3 \pm 0.2 \times 10^{17}$ \\
\hline $\mathrm{HDC}^{18} \mathrm{O}$ & $\leq 1.4 \pm 0.4 \times 10^{14}$ \\
\hline $\mathrm{D}_{2} \mathrm{CO}$ & $1.6_{-0.5}^{+0.4} \times 10^{16}$ \\
\hline $\mathrm{D}_{2}{ }^{13} \mathrm{CO}$ & $2.2_{-0.4}^{+0.2} \times 10^{14}$ \\
\hline
\end{tabular}

Table 2. Derived fractionation ratios for the various forms of formaldehyde.

\begin{tabular}{llll}
\hline \hline Species & Ratio & Comb. $^{a}$ & $\mathrm{D} / \mathrm{H}$ \\
\hline $\mathrm{HDCO} / \mathrm{H}_{2} \mathrm{CO}$ & $6.8_{-1.3}^{+1.1} \%$ & $6.5 \pm 1 \%$ & $3.25 \pm 1 \%$ \\
$\mathrm{HDC}{ }^{18} \mathrm{O} / \mathrm{H}_{2} \mathrm{C}^{18} \mathrm{O}$ & $6.0 \pm 1.5 \%$ & - & \\
$\mathrm{D}_{2} \mathrm{CO} / \mathrm{HDCO}$ & $12.8_{-4.1}^{+3.3} \%$ & & $25.6_{-4.1}^{+3.3} \%$ \\
$\mathrm{D}_{2} \mathrm{CO} / \mathrm{H}_{2} \mathrm{CO}$ & $0.9_{-0.3}^{+0.2} \%$ & $0.6(4) \pm 0.1 \%$ & $8.0 \pm 0.1 \%$ \\
$\mathrm{D}_{2}{ }^{13} \mathrm{CO} / \mathrm{H}_{2}{ }^{13} \mathrm{CO}$ & $0.6_{-0.2}^{+0.1} \%$ & - & \\
\hline $\mathrm{H}_{2} \mathrm{CO} / \mathrm{H}_{2}{ }^{13} \mathrm{CO}$ & $52_{-16}^{+10}$ & $56_{-11}^{+8}$ & \\
$\mathrm{D}_{2} \mathrm{CO} / \mathrm{D}_{2}{ }^{13} \mathrm{CO}$ & $74_{-24}^{+18}$ & - & \\
\hline $\mathrm{H}_{2} \mathrm{CO} / \mathrm{H}_{2} \mathrm{C}^{18} \mathrm{O}$ & $800_{-98}^{+47}$ & $805_{-79}^{+43}$ & \\
$\mathrm{HDCO} / \mathrm{HDC}^{18} \mathrm{O}$ & $908 \pm 263$ & - & \\
\hline $\mathrm{H}_{2} \mathrm{CO} / \mathrm{H}_{2} \mathrm{C}^{17} \mathrm{O}$ & $2596_{-395}^{+639}$ & & \\
\hline $\mathrm{H}_{2} \mathrm{C}{ }^{18} \mathrm{O} / \mathrm{H}_{2} \mathrm{C}^{17} \mathrm{O}$ & $3.2_{-0.3}^{+0.2}$ & & \\
\hline
\end{tabular}

Notes. The errors are propagated from the uncertainty in the determined column densities. ${ }^{(a)}$ Combined isotopologue ratio (following Barlow 2003).

increases the constraint. Thus, a wide spread in $E_{\mathrm{u}}$ was used to constrain the $T_{\text {ex }}$, but avoiding the $11_{1,10}-11_{1,11}$ line at $346.74 \mathrm{GHz}$ which is optically thick.

\subsection{Column densities and ratios}

The best fit column densities at $T_{\mathrm{ex}}=106 \pm 13 \mathrm{~K}$ for the species are listed in Table 1. The uncertainty in abundance is dominated by the uncertainty in excitation temperature. In Table 2 the various column density ratios for the studied isotopologues are shown. Ratios where multiple estimates exist using isotopologues, that is, $\mathrm{HDCO} / \mathrm{H}_{2} \mathrm{CO}$ and $\mathrm{HDC}^{18} \mathrm{O} / \mathrm{H}_{2} \mathrm{C}^{18} \mathrm{O}$ for the $\mathrm{HDCO} / \mathrm{H}_{2} \mathrm{CO}$ ratio, are combined. When combining values with asymmetric uncertainties care has to be taken when calculating the best combined estimate (for a detailed discussion and solution see Sect. 3 in Barlow 2003). To constrain the column density of the main isotopologue $\mathrm{H}_{2} \mathrm{CO}$ only one detection and one upper-limit (non-detection) are used in the fit. The other $\mathrm{H}_{2} \mathrm{CO}$ transitions are optically thick, by a large margin, and they also show some extended emission (van der Wiel, in prep.).

\section{Discussion}

\subsection{Column densities and excitation temperature}

The derived excitation temperature of $106 \pm 13 \mathrm{~K}$ agrees with the results for some of the other molecules studied within the PILS 
survey. Acetaldehyde, ketene, ethylene oxide, acetone, propanal, and dimethyl ether show a similar excitation temperature, on the order of $125 \mathrm{~K}$ (Lykke et al. 2017; Jørgensen et al. 2017). Interestingly van Dishoeck et al. (1995) derived, after subtracting the extended contribution from the central emission, a kinetic temperature of $100_{-20}^{+40} \mathrm{~K}$ from the $\mathrm{H}_{2} \mathrm{CO} 3_{22}-3_{21} / 3_{03}-2_{02}$ ratio toward IRAS 16293-2422.

The derived column densities fit the observed spectrum well using the assumed $T_{\mathrm{ex}}$ of $106 \mathrm{~K}$ from $\mathrm{HDCO}$. The $\mathrm{H}_{2}{ }^{13} \mathrm{CO}$ line at $355.04 \mathrm{GHz}$ is partly blended with the weaker counterpart of $\mathrm{H}_{2} \mathrm{C}^{17} \mathrm{O}$. This means that the $\mathrm{H}_{2}{ }^{13} \mathrm{CO}$ column density might be overestimated by a small amount, however the effect is within the uncertainties. $\mathrm{A}_{2} \mathrm{C}^{17} \mathrm{O}$ column density and excitation temperature taken at the lower uncertainty limit will make its blending effect on $\mathrm{H}_{2}{ }^{13} \mathrm{CO}$ insignificant.

\subsection{Deuterium fractionation}

In this section we present the deuterium fractionation calculated from the observations and then compare these to the model of Taquet et al. (2014).

\subsubsection{Measured $\mathrm{D} / \mathrm{H}$ ratios}

Together with the column density ratios for the studied isotopologues in Table 2 are the best estimated D/H ratios as well (combined measurements where applicable), where $\mathrm{D} / \mathrm{H}$ is given by $\mathrm{HDCO} / \mathrm{H}_{2} \mathrm{CO} \times 0.5, \mathrm{D}_{2} \mathrm{CO} / \mathrm{HDCO} \times 2$, and $\sqrt{\mathrm{D}_{2} \mathrm{CO} / \mathrm{H}_{2} \mathrm{CO}}$. While the errors quoted for the column densities are derived by fitting spectra with the column density for the highest and lowest excitation temperature, the errors in the ratios were derived with error propagation. Thus the uncertainties of the column densities and in extension the fractionation ratios, reflect also the uncertainty in excitation temperature.

The $\mathrm{HDC}^{18} \mathrm{O} / \mathrm{H}_{2} \mathrm{C}^{18} \mathrm{O}$ ratio is $6.0 \%(\mathrm{D} / \mathrm{H}$ ratio of $3.0 \%)$, and it agrees with the main isotopologues $\mathrm{HDCO} / \mathrm{H}_{2} \mathrm{CO}$ ratio of $6.8 \%(\mathrm{D} / \mathrm{H}$ ratio of $3.4 \%)$, confirming the method used for constraining the column densities. Their combined ratio $6.5 \%(\mathrm{D} / \mathrm{H}$ ratio $3.25 \%$ ) is taken as the best estimate. The $\mathrm{D}_{2}{ }^{13} \mathrm{CO} / \mathrm{H}_{2}{ }^{13} \mathrm{CO}$ ratio $(0.6 \%)$ is also similar to the main form $\mathrm{D}_{2} \mathrm{CO} / \mathrm{H}_{2} \mathrm{CO}$ ratio $(0.9 \%)$, their combined ratio being $0.6(4) \%(\mathrm{D} / \mathrm{H}$ ratio $8.0 \%)$. Finally, the $\mathrm{D}_{2} \mathrm{CO} / \mathrm{HDCO}$ ratio is $12.8 \%$ (D/H ratio is $25.6 \%$ ).

These numbers show that the $\mathrm{D}_{2} \mathrm{CO} / \mathrm{HDCO}$ ratio is two times higher than the $\mathrm{HDCO} / \mathrm{H}_{2} \mathrm{CO}$ ratio, significantly more than the $1 / 4$ expected from statistical arguments (see Eq. (1) and related text). Furthermore, other organics show $\mathrm{D} / \mathrm{H}$ ratios similar to this (Jørgensen et al. 2017). This shows that for a full understanding a more complex interpretation than simple statistical arguments is needed. Whether other protostars show similar deuterium fractionation ratios for $\mathrm{H}_{2} \mathrm{CO}$ as deduced here is not yet clear, observations of more protostars are needed to investigate what role initial environment and evolution might play in determining the ratios.

\subsubsection{Models}

Taquet et al. (2012) and more recently Taquet et al. (2014) studied the evolution of deuterated ices during the earliest stages of star formation, including the prestellar and protostellar (Class 0) phase. In the 2012 study the importance of abstraction and substitution reactions is shown, as also supported by lab data (Hidaka et al. 2009). Furthermore, the $\mathrm{HDCO} / \mathrm{H}_{2} \mathrm{CO}$ ratio is not affected by the inclusion of abstraction reactions due to the efficiency of the deuterium abstraction on HDCO, forming back $\mathrm{H}_{2} \mathrm{CO}$. Thus, constraining the deuterium fractionation in all forms of formaldehyde, especially the doubly deuterated form is crucial for constraining the formation conditions and the chemical models. The results show that a reaction network without abstraction and substitution reactions cannot reproduce the $\mathrm{HDCO} / \mathrm{H}_{2} \mathrm{CO}$ and $\mathrm{D}_{2} \mathrm{CO} / \mathrm{HDCO}$ ratios simultaneously. Including abstraction reactions shows that the ratios are reproducible at moderate densities $\left(n_{\mathrm{H}}=10^{6} \mathrm{~cm}^{-3}\right)$. The model in the 2014 study traces the density and temperature evolution from a (inside-out) collapsing core to the end of the deeply-embedded stage (Class 0 ). The observed (combined, best estimate) ratios of $\mathrm{HDCO} / \mathrm{H}_{2} \mathrm{CO}$ of $6.5 \pm 1 \%$ and $\mathrm{D}_{2} \mathrm{CO} / \mathrm{H}_{2} \mathrm{CO}$ of $0.6(4) \pm 0.1 \%$ are best reproduced by the model at $t=1.1 \times 10^{5}$ years. This timescale represents the beginning of the Class 0 stage, where the $\mathrm{D} / \mathrm{H}$ ratio in $\mathrm{HDCO}$ is $4.1 \%$ and $0.18 \%$ for $\mathrm{D}_{2} \mathrm{CO}$ (Table 7 in Taquet et al. 2014). While the density and temperature profile at this time step from Taquet et al. (2014) is similar to the physical model of Crimier et al. (2010) for IRAS 16293-2422 specifically, the modeled deuterium fractionation observed in the hot corino gas is mostly due to the deuteration processses of formaldehyde in ices in the previous dense cloud stage and thus less dependent on the current physical structure. In Taquet et al. (2014) the assumed size of the beam in the observations of warm gas with interferometers is 0 ".4 (or $50 \mathrm{AU}$ ) which is very similar to the beam in this study $\left(00^{\prime \prime 5}\right)$. The excitation temperature derived shows that we are probing the warm gas of the hot corino. The ratios derived using single dish telescopes, which may apply to larger scales, are more difficult to compare with the model results. It is not clear to what extent opacity effects, assumed size of emitting region and contributions of various emission components play a role in the previous single dish observations, thus we refrain from any extensive comparison with those results.

The main production phase of deuterated molecules comes toward the end of the prestellar core stage (Furuya et al. 2016). This is in agreement with previous studies of starless cores using $\mathrm{N}_{2} \mathrm{H}^{+}$and $\mathrm{N}_{2} \mathrm{D}^{+}$(Crapsi et al. 2005). Taquet et al. (2014) also trace the deuteration through these early stages, with similar conclusions. The model reproduces the large differences between singly and doubly deuterated forms with the $\mathrm{D}_{2} \mathrm{O} / \mathrm{HDO}$ ratio being about seven times higher than the $\mathrm{HDO} / \mathrm{H}_{2} \mathrm{O}$ ratio. In formaldehyde toward IRAS $16293-2422$ the $\mathrm{D}_{2} \mathrm{CO} / \mathrm{HDCO}$ ratio is about $2 \times \mathrm{HDCO} / \mathrm{H}_{2} \mathrm{CO}$, a similar but much less pronounced effect than for water.

\subsubsection{Formaldehyde and methanol}

Methanol $\left(\mathrm{CH}_{3} \mathrm{OH}\right)$ was studied in the framework of the ALMAPILS survey (Jørgensen et al. 2017). The deuterium fractionation $(\mathrm{D} / \mathrm{H})$ derived toward IRAS 16293-2422 B in methanol is $2 \%$. This is comparable to singly deuterated formaldehyde. Interesting enough, the time scale of the astrochemical model presented by Taquet et al. (2014) that best reproduces this level of fractionation in methanol is also $t=1.1 \times 10^{5}$ years. This could indicate that IRAS $16293-2422$ B is indeed a very young Class 0 source. Using the column density derived for methanol by Jørgensen et al. the $\mathrm{H}_{2} \mathrm{CO} / \mathrm{CH}_{3} \mathrm{OH}$ ratio is 0.19 toward IRAS 16293-2422 B. A ratio of one was inferred toward the inner regions of the same source by Maret et al. (2005) using single dish observations and radiative transfer modeling, clearly different from the ratio presented in this study. However, the large uncertainties inherent in using single dish measurements to constrain inner envelope abundances makes a comparison difficult. The measured $\mathrm{H}_{2} \mathrm{CO} / \mathrm{CH}_{3} \mathrm{OH}$ ratio of 0.19 is close to what has been constrained in ices toward protostars, ranging from 0.1 to 0.67 (Keane et al. 2001). This strengthens the view that warm gas in the innermost regions of protostars represent the bulk ice composition. The measured $\mathrm{H}_{2} \mathrm{CO} / \mathrm{CH}_{3} \mathrm{OH}$ ratio is in agreement 
with what was measured in hot cores by Bisschop et al. (2007), where the mean and $1 \sigma$ standard deviation presented toward seven hot cores was $0.22 \pm 0.05$.

\subsection{Other isotopic ratios}

While the ${ }^{16} \mathrm{O} /{ }^{18} \mathrm{O}$ ratio is 500 at this distance from the Galactic center (Wilson \& Rood 1994; Wilson 1999), the ratio measured in formaldehyde for IRAS $16293-2422 \mathrm{~B}$ is 800 for $\mathrm{H}_{2} \mathrm{CO}$ and 908 for HDCO, both slightly higher than expected at these distances from the Galactic center. The combined ratio for the two measurements is $805_{-79}^{+43}$. Given the uncertainties involved, including the Galactic gradient, ${ }^{16} \mathrm{O} /{ }^{18} \mathrm{O}=400-600$ at $8 \mathrm{kpc}$, it is difficult to draw any conclusions, although the combined best estimate ratio is higher than the range in the local ISM.

The ${ }^{18} \mathrm{O} /{ }^{17} \mathrm{O}$ ratio in formaldehyde toward IRAS $16293-2422 \mathrm{~B}$ is $3.2_{-0.3}^{+0.2}\left(\mathrm{H}_{2} \mathrm{C}^{18} \mathrm{O} / \mathrm{H}_{2} \mathrm{C}^{17} \mathrm{O}\right)$. Upper limits of the isotopic variant $\mathrm{HDC}^{17} \mathrm{O}$ derived using the $6_{1,6}-5_{1,5}$ transition at $360.962 \mathrm{GHz}$ gives a ${ }^{18} \mathrm{O} /{ }^{17} \mathrm{O}$ ratio of two or more, compatible with the local ISM value and $\mathrm{H}_{2} \mathrm{C}^{18} \mathrm{O} / \mathrm{H}_{2} \mathrm{C}^{17} \mathrm{O}$ ratios. Jørgensen et al. (2002) measured the $\mathrm{C}^{18} \mathrm{O} / \mathrm{C}^{17} \mathrm{O}$ ratio toward the envelopes of 19 protostars, including Class 0 and I sources. Toward IRAS 16293-2422 the measured ratio was 3.9 , in agreement with the ratio measured for formaldehyde, and roughly $74 \%$ of all the measured sources showed a ratio of between two and four. The $\mathrm{H}_{2} \mathrm{C}^{18} \mathrm{O} / \mathrm{H}_{2} \mathrm{C}^{17} \mathrm{O}$ ratio was first measured toward the Galactic center, $4 \mathrm{kpc}$ molecular ring and the local ISM to be $3.2 \pm 0.2$ (Penzias 1981). Wouterloot et al. (2008) discovered a tentative gradient in the ${ }^{18} \mathrm{O} /{ }^{17} \mathrm{O}$ ratio with galactocentric distance ranging from three in the center to five in the outer galaxy, and more recently Li et al. (2016, for example, Fig. 3) strengthened this conclusion. The ratio in the outermost galaxy, beyond $11 \mathrm{kpc}$ is still uncertain. The observed gradient is consistent with an inside-out formation of the galaxy. The observed value toward IRAS 16293-2422 fits in the galactic ${ }^{18} \mathrm{O} /{ }^{17} \mathrm{O}$ gradient and could indicate a fairly normal formation environment at this distance from the Galactic center. The deviating ${ }^{16} \mathrm{O} /{ }^{18} \mathrm{O}$ ratio (see previous paragraph) is not fully consistent with this conclusion.

The ${ }^{12} \mathrm{C} /{ }^{13} \mathrm{C}$ ratio for $\mathrm{H}_{2} \mathrm{CO}$ and $\mathrm{D}_{2} \mathrm{CO}$ and their combined ratio of $56_{-11}^{+8}$ are consistent with the ratio of $68 \pm 30$, which is relevant for these galactocentric distances (Wilson \& Rood 1994; Wilson 1999; Milam et al. 2005). While lines of the isotopic variant $\mathrm{HD}^{13} \mathrm{CO}$ are too weak to detect in our survey, its upper limit is compatible with a ${ }^{12} \mathrm{C} /{ }^{13} \mathrm{C}$ ratio of $\geq 55$ (using mainly the $11_{1,10}-11_{1,11}$ transition). Recent results from the ALMA-PILS survey presented in Jørgensen et al. (2017) show that the ${ }^{12} \mathrm{C} /{ }^{13} \mathrm{C}$ ratio in glycolaldehyde, ethanol, methyl formate, and dimethyl ether is around 20-40, lower than for formaldehyde, methanol and the surrounding ISM. This is attributed to the later formation of the low ${ }^{12} \mathrm{C} /{ }^{13} \mathrm{C}$ ratio species, when the availability of ${ }^{13} \mathrm{C}$ is higher.

\subsection{The path to more complex molecules}

Since formaldehyde is thought to play a role in the formation of more complex molecules, it is interesting to compare the deuteration between formaldehyde and its potential daughter species. Formaldehyde may also lead to the formation of formamide $\left(\mathrm{NH}_{2} \mathrm{CHO}\right)$ on grain surfaces as shown by some laboratory experiments (Fedoseev et al. 2016). Barone et al. (2015) proposed that formamide could form due to the reaction between $\mathrm{H}_{2} \mathrm{CO}$ and $\mathrm{NH}_{2}$ in the gas phase. Recently Coutens et al. (2016) detected the mono-deuterated forms of formamide using the ALMA-PILS survey and reported a deuteration level of $\sim 2 \%$ assuming a standard ${ }^{12} \mathrm{C} /{ }^{13} \mathrm{C}$ ratio of 68 . Skouteris et al. (2017) also calculated the rate coefficients of the reactions producing deuterated formamide. Consequently, a comparison of the deuteration of formamide and formaldehyde can help determine if this gas phase pathway (for formamide) is possible or if it has to be ruled out in this source. According to the respective rate coefficients determined for $\mathrm{NH}_{2} \mathrm{CHO}$ and $\mathrm{NH}_{2} \mathrm{CDO}$ by Skouteris et al. (2017), the $\mathrm{HDCO} / \mathrm{H}_{2} \mathrm{CO}$ ratio should be three times higher than the $\mathrm{NH}_{2} \mathrm{CDO} / \mathrm{NH}_{2} \mathrm{CHO}$ ratio if this gasphase reaction occurs. At similar spatial scales, the best estimate $\mathrm{HDCO} / \mathrm{H}_{2} \mathrm{CO}$ ratio is $6.5 \pm 1 \%$, that is approximately three times higher than the $\mathrm{NH}_{2} \mathrm{CDO} / \mathrm{NH}_{2} \mathrm{CHO}$ ratio. Thus, a gas phase formation pathway for formamide cannot be ruled out. Since the expected deuteration levels for the case of a grain surface formation pathway has not been explored, none of the scenarios for formamide formation can be ruled out from this comparison at this point.

\section{Summary and outlook}

Measuring the deuterium fractionation is an important tool in understanding the early chemical evolution of forming protostars. The evolving picture is that the gas phase deuteration of molecules in the inner warm regions, representative of the bulk ice, seems to be lower than previously thought based on single dish observations. This highlights that more high-sensitivity and -resolution radio interferometric observations are needed to probe this gas reservoir and unlock the chemical history and future of deeply embedded low-mass protostars.

In this study we have detected several lines of various isotopologues of formaldehyde toward the deeply embedded lowmass protostar IRAS 16293-2422 B as part of the ALMA-PILS survey. Both $\mathrm{H}_{2} \mathrm{C}^{17} \mathrm{O}$ and $\mathrm{D}_{2}{ }^{13} \mathrm{CO}$ are detected for the first time in the ISM. Many of the lines are optically thick due to the high densities probed. The determined excitation temperature for HDCO is $106 \pm 13 \mathrm{~K}$, similar to several other molecules in the same survey, and previous measurements.

Assuming the same excitation temperature constrained from HDCO for all the forms of formaldehyde to determine the column densities shows that many of the transitions are optically thick and abundances high. The detected forms of formaldehyde are: $\mathrm{H}_{2} \mathrm{CO}, \mathrm{H}_{2}{ }^{13} \mathrm{CO}, \mathrm{H}_{2} \mathrm{C}^{17} \mathrm{O}, \mathrm{H}_{2} \mathrm{C}^{18} \mathrm{O}, \mathrm{HDCO}, \mathrm{HDC}^{18} \mathrm{O}$, $\mathrm{D}_{2} \mathrm{CO}$, and $\mathrm{D}_{2}{ }^{13} \mathrm{CO}$. The large number of lines covered by the unbiased spectral survey makes it possible to constrain the column density by focusing on the optically thin, unblended lines.

The measured $\mathrm{HDCO} / \mathrm{H}_{2} \mathrm{CO}$ ratio is $6.5 \pm 1 \%$. This is significantly lower than what was previously estimated using single dish telescopes $(14-33 \%)$. This is also true for doubly deuterated formaldehyde, where the $\mathrm{D}_{2} \mathrm{CO} / \mathrm{HDCO}$ ratio is $12.8_{-4.1}^{+3.3} \%$, and $\mathrm{D}_{2} \mathrm{CO} / \mathrm{H}_{2} \mathrm{CO}$ ratio is $0.6 \pm 0.1 \%$. The lines observed with single dish telescopes in previous studies also covered by the ALMA-PILS survey are significantly optically thick. The source size is better constrained here by spatially resolving the emitting region. These effects could be the reason for the different ratio(s).

These levels of deuterium fractionation are in line with formaldehyde forming on the grains once $\mathrm{CO}$ has frozen onto the surface as soon as the temperature drops low enough. The astrochemical model of Taquet et al. (2014) can approximately reproduce the deuterium fractionation observed in formaldehyde with a grain surface formation pathway dominating the production. However, the role of gas phase formation routes is still not clear. 
The effect seen for water (Persson et al. 2013; Coutens et al. 2012; Furuya et al. 2016), where $\mathrm{D}_{2} \mathrm{O} / \mathrm{HDO}$ is significantly higher than the $\mathrm{HDO} / \mathrm{H}_{2} \mathrm{O}$ ratio is only minor for formaldehyde due to the even later start of formation toward the end of the prestellar core phase in comparison to water. This also implies higher absolute deuterium fractionation $\left(\mathrm{D} / \mathrm{H}_{\mathrm{H}_{2} \mathrm{CO}} \sim 3 \%\right.$, $\mathrm{D} / \mathrm{H}_{\mathrm{H}_{2} \mathrm{O}} \sim 0.1 \%$ ), due to the more favorable conditions (low o/pratio, low $T$, higher accretion of $\mathrm{D}$ atoms onto grains). The deuterium fractionation presented for formaldehyde is similar to that of other molecules with similar complexity such as methanol $\left(\mathrm{CH}_{3} \mathrm{OH}\right.$; Jørgensen et al. 2017) and formamide $\left(\mathrm{NH}_{2} \mathrm{CHO}\right.$; Coutens et al. 2016), at around 2-3\%. This formation-time dependence on deuteration implies that more complex molecules which form even later should have even higher levels of deuterium fractionation.

The ${ }^{12} \mathrm{C} /{ }^{13} \mathrm{C}$ and ${ }^{18} \mathrm{O} /{ }^{17} \mathrm{O}$ ratios in formaldehyde are consistent with the values measured for the surrounding ISM at the relevant galactocentric distances. This could indicate a relatively common formation environment in the Galaxy. However, the ${ }^{16} \mathrm{O} /{ }^{18} \mathrm{O}$ ratio is slightly higher than the local ISM value thus it is not possible to draw any strong conclusions based on this.

Future high resolution interferometric observations constraining the deuterium fractionation in formaldehyde of other sources, could shed light on any possible dependence on environment and mass. In addition to this, multi transition single dish observations unambiguously characterizing the cold gas would improve the global picture of formaldehyde deuterium fractionation.

Acknowledgements. This research made use of Astropy and Astroquery, community-developed Python packages for Astronomy (Astropy Collaboration et al. 2013; Ginsburg et al. 2017). M.V.P. postdoctoral position is funded by the ERC consolidator grant 614264. J.K.J. acknowledges support from the European Research Council (ERC) under the European Union's Horizon 2020 research and innovation programme (grant agreement No. 646908) through ERC Consolidator Grant "S4F". Research at Centre for Star and Planet Formation is funded by the Danish National Research Foundation. A.C.'s postdoctoral grant is funded by the ERC Starting Grant 3DICE (grant agreement 336474). V.T. has received funding from the European Union's Horizon 2020 research and innovation programme under the Marie Skłodowska-Curie grant agreement No. 664931. E.v.D. acknowledges EU AERC grant 291141 CHEMPLAN and a KNAW professorship prize. This paper makes use of the following ALMA data: ADS/JAO.ALMA\#2013.1.00278.S ALMA is a partnership of ESO (representing its member states), NSF (USA) and NINS (Japan), together with NRC (Canada) and NSC and ASIAA (Taiwan) in cooperation with the Republic of Chile. The Joint ALMA Observatory is operated by ESO, AUI/NRAO and NAOJ.

\section{References}

Astropy Collaboration, Robitaille, T. P., Tollerud, E. J., et al. 2013, A\&A, 558 A33

Avni, Y. 1976, ApJ, 210, 642

Barlow, R. 2003, in Statistical Problems in Particle Physics, Astrophysics, and Cosmology, eds. L. Lyons, R. Mount, \& R. Reitmeyer, 250

Barone, V., Latouche, C., Skouteris, D., et al. 2015, MNRAS, 453, L31

Bisschop, S. E., Jørgensen, J. K., van Dishoeck, E. F., \& de Wachter, E. B. M. 2007, A\&A, 465, 913

Bisschop, S. E., Jørgensen, J. K., Bourke, T. L., Bottinelli, S., \& van Dishoeck, E. F. 2008, A\&A, 488, 959

Bocquet, R., Demaison, J., Poteau, L., et al. 1996, J. Mol. Spectr., 177, 154

Bocquet, R., Demaison, J., Cosléou, J., et al. 1999, J. Mol. Spectr., 195, 345

Bottinelli, S., Ceccarelli, C., Neri, R., et al. 2004, ApJ, 617, L69

Brünken, S., Müller, H. S. P., Lewen, F., \& Winnewisser, G. 2003, Phys. Chem. Chem. Phys. (Incorporating Faraday Transactions), 5

Ceccarelli, C., Castets, A., Loinard, L., Caux, E., \& Tielens, A. G. G. M. 1998, A\&A, 338, L43

Ceccarelli, C., Loinard, L., Castets, A., et al. 2001, A\&A, 372, 998

Charnley, S. B., Tielens, A. G. G. M., \& Rodgers, S. D. 1997, ApJ, 482, L203

Cornet, R. A., \& Winnewisser, G. 1980a, J. Mol. Spectr., 80, 438

Cornet, R. A., Landsberg, B. M., \& Winnewisser, G. 1980b, J. Mol. Spectr., 82, 253
Coutens, A., Vastel, C., Caux, E., et al. 2012, A\&A, 539, A132

Coutens, A., Jørgensen, J. K., Persson, M. V., et al. 2014, ApJ, 792, L5

Coutens, A., Jørgensen, J. K., van der Wiel, M. H. D., et al. 2016, A\&A, 590, L6

Crapsi, A., Caselli, P., Walmsley, C. M., et al. 2005, ApJ, 619, 379

Crimier, N., Ceccarelli, C., Maret, S., et al. 2010, A\&A, 519, A65

Dangoisse, D., Willemot, E., \& Bellet, J. 1978, J. Mol. Spectr., 71, 414

Fedoseev, G., Chuang, K.-J., van Dishoeck, E. F., Ioppolo, S., \& Linnartz, H. 2016, MNRAS, 460, 4297

Flygare, W. H., \& Lowe, J. T. 1965, J. Chem. Phys., 43, 3645

Fuchs, G. W., Cuppen, H. M., Ioppolo, S., et al. 2009, A\&A, 505, 629

Furuya, K., Aikawa, Y., Hincelin, U., et al. 2015, A\&A, 584, A124

Furuya, K., van Dishoeck, E. F., \& Aikawa, Y. 2016, A\&A, 586, A127

Ginsburg, A., Parikh, M., Woillez, J., et al. 2017, Astrophysics Source Code Library [record ascl: 1708.004]

Hidaka, H., Watanabe, M., Kouchi, A., \& Watanabe, N. 2009, ApJ, 702, 291

Isokoski, K., Bottinelli, S., \& van Dishoeck, E. F. 2013, A\&A, 554, A100

Jørgensen, J. K., Schöier, F. L., \& van Dishoeck, E. F. 2002, A\&A, 389, 908

Jørgensen, J. K., Bourke, T. L., Nguyen Luong, Q., \& Takakuwa, S. 2011, A\&A, 534, A100

Jørgensen, J. K., Favre, C., Bisschop, S. E., et al. 2012, ApJ, 757, L4

Jørgensen, J. K., van der Wiel, M. H. D., Coutens, A., et al. 2016, A\&A, 595, A117

Jørgensen, J. K., Müller, H. S. P., Calcutt, H., et al. 2017, A\&A, submitted

Keane, J. V., Tielens, A. G. G. M., Boogert, A. C. A., Schutte, W. A., \& Whittet, D. C. B. 2001, A\&A, 376, 254

Kuan, Y.-J., Huang, H.-C., Charnley, S. B., et al. 2004, ApJ, 616, L27

Li, H.-K., Zhang, J.-S., Liu, Z.-W., et al. 2016, Res. Astron. Astrophys., 16, 47

Ligterink, N. F. W., Coutens, A., Kofman, V., et al. 2017, MNRAS, 469, 2219

Lohilahti, J., \& Horneman, V.-M. 2004, J. Mol. Spectr., 228, 1

Lohilahti, J., Mattila, H., Horneman, V.-M., \& Pawłowski, F. 2005, J. Mol. Spectr., 234, 279

Loinard, L., Castets, A., Ceccarelli, C., et al. 2000, A\&A, 359, 1169

Loinard, L., Torres, R. M., Mioduszewski, A. J., \& Rodríguez, L. F. 2008, ApJ, 675, L29

Lykke, J. M., Coutens, A., Jørgensen, J. K., et al. 2017, A\&A, 597, A53

Maret, S., Ceccarelli, C., Tielens, A. G. G. M., et al. 2005, A\&A, 442, 527

Martín-Doménech, R., Rivilla, V. M., Jiménez-Serra, I., et al. 2017, MNRAS, 469, 2230

Milam, S. N., Savage, C., Brewster, M. A., Ziurys, L. M., \& Wyckoff, S. 2005, ApJ, 634, 1126

Müller, H. S. P., \& Lewen, F. 2017, J. Mol. Spectr., 331, 28

Müller, H. S. P., Winnewisser, G., Demaison, J., Perrin, A., \& Valentin, A. 2000a, J. Mol. Spectr., 200, 143

Müller, H. S. P., Gendriesch, R., Lewen, F., \& Winnewisser, G. 2000b, Zeitschrift Naturforschung Teil A, 55, 486

Müller, H. S. P., Gendriesch, R., Margulès, L., et al. 2000c, Phys. Chem. Chem. Phys., 2, 3401

Müller, H. S. P., Thorwirth, S., Roth, D. A., \& Winnewisser, G. 2001, A\&A, 370, L49

Müller, H. S. P., Schlöder, F., Stutzki, J., \& Winnewisser, G. 2005, J. Mol. Struct., 742,215

Pagani, L., Salez, M., \& Wannier, P. G. 1992, A\&A, 258, 479

Parise, B., Ceccarelli, C., Tielens, A. G. G. M., et al. 2006, A\&A, 453, 949

Penzias, A. A. 1981, ApJ, 249, 518

Persson, M. V., Jørgensen, J. K., \& van Dishoeck, E. F. 2013, A\&A, 549, L3

Persson, M. V., Jørgensen, J. K., van Dishoeck, E. F., \& Harsono, D. 2014, A\&A, 563, A74

Press, W. H., Teukolsky, S. A., Vetterling, W. T., \& Flannery, B. P. 2002, Numerical recipes in $\mathrm{C}++$ : the art of scientific computing (CUP)

Prodanović, T., Steigman, G., \& Fields, B. D. 2010, MNRAS, 406, 1108

Roberts, H., \& Millar, T. J. 2000, A\&A, 361, 388

Roberts, H., Herbst, E., \& Millar, T. J. 2004, A\&A, 424, 905

Schöier, F. L., Jørgensen, J. K., van Dishoeck, E. F., \& Blake, G. A. 2004, A\&A, 418,185

Skouteris, D., Vazart, F., Ceccarelli, C., et al. 2017, MNRAS, 468, L1

Taquet, V., Ceccarelli, C., \& Kahane, C. 2012, ApJ, 748, L3

Taquet, V., Charnley, S. B., \& Sipilä, O. 2014, ApJ, 791, 1

Tielens, A. G. G. M. 1983, A\&A, 119, 177

Turner, B. E. 1990, ApJ, 362, L29

van Dishoeck, E. F., Blake, G. A., Jansen, D. J., \& Groesbeck, T. D. 1995, ApJ, 447,760

Watanabe, N., \& Kouchi, A. 2002, ApJ, 571, L173

Wilson, T. L. 1999, Rep. Prog. Phys., 62, 143

Wilson, T. L., \& Rood, R. 1994, ARA\&A, 32, 191

Wouterloot, J. G. A., Henkel, C., Brand, J., \& Davis, G. R. 2008, A\&A, 487, 237

Zakharenko, O., Motiyenko, R. A., Margulès, L., \& Huet, T. R. 2015, J. Mol. Spectr., 317, 41 


\section{Appendix A: Formaldehyde lines used in the analysis}

Table A.1. Lines detected in the ALMA-PILS spectrum of IRAS 16293-2422.

\begin{tabular}{|c|c|c|c|c|c|c|}
\hline Species & $\begin{array}{c}Q_{1} \\
J_{K_{A} K_{B}}\end{array}$ & $Q_{2}$ & $\begin{array}{c}v \\
\mathrm{GHz}\end{array}$ & $\begin{array}{l}A_{i j} \\
\log _{10}()\end{array}$ & $\begin{array}{r}E_{\mathrm{u}} \\
\mathrm{K}\end{array}$ & Fit? \\
\hline \multirow[t]{3}{*}{$\mathrm{H}_{2} \mathrm{CO}$} & $5_{1,5}$ & $4_{1,4}$ & 351.769 & -2.9201 & 62.5 & \\
\hline & $8_{2,6}$ & 90,9 & 361.968 & -5.9856 & 173.5 & घ \\
\hline & $5_{0,5}$ & $4_{0,4}$ & 362.736 & -2.8626 & 52.3 & \\
\hline \multirow[t]{8}{*}{$\mathrm{H}_{2} \mathrm{C}^{17} \mathrm{O}$} & $5_{1,5}$ & $4_{1,4}$ & 343.333 & -2.9518 & 61.3 & \\
\hline & $5_{0,5}$ & $4_{0,4}$ & 353.820 & -2.8950 & 51.0 & घ \\
\hline & $5_{2,4}$ & $4_{2,3}$ & 354.908 & -2.9665 & 98.4 & घ \\
\hline & $5_{4,2}$ & $4_{4,1}$ & 355.042 & -3.3340 & 240.1 & \\
\hline & $5_{4,1}$ & $4_{4,0}$ & 355.042 & -3.3340 & 240.1 & \\
\hline & $5_{3,3}$ & $4_{3,2}$ & 355.202 & -3.0835 & 157.5 & \\
\hline & $5_{3,2}$ & $4_{3,1}$ & 355.214 & -3.0834 & 157.5 & घ \\
\hline & $5_{2,3}$ & $4_{2,2}$ & 356.187 & -2.9619 & 98.5 & घ \\
\hline \multirow[t]{9}{*}{$\mathrm{H}_{2} \mathrm{C}^{18} \mathrm{O}$} & $5_{1,5}$ & $4_{1,4}$ & 335.816 & -2.9805 & 60.2 & \\
\hline & $5_{0,5}$ & $4_{0,4}$ & 345.881 & -2.9246 & 49.9 & \\
\hline & $5_{2,4}$ & $4_{2,3}$ & 346.869 & -2.9963 & 97.4 & घ \\
\hline & $5_{4,2}$ & $4_{4,1}$ & 346.984 & -3.3639 & 239.6 & a \\
\hline & $5_{4,1}$ & $4_{4,0}$ & 346.984 & -3.3639 & 239.6 & घ \\
\hline & $5_{3,3}$ & $4_{3,2}$ & 347.134 & -3.1134 & 156.7 & \\
\hline & $5_{3,2}$ & $4_{3,1}$ & 347.144 & -3.1134 & 156.7 & \\
\hline & $5_{2,3}$ & $4_{2,2}$ & 348.032 & -2.9920 & 97.5 & a \\
\hline & $5_{1,4}$ & $4_{1,3}$ & 357.741 & -2.8982 & 63.4 & \\
\hline \multirow[t]{7}{*}{$\mathrm{H}_{2}{ }^{13} \mathrm{CO}$} & $5_{1,5}$ & $4_{1,4}$ & 343.326 & -2.9517 & 61.3 & \\
\hline & $5_{0,5}$ & $4_{0,4}$ & 353.812 & -2.8949 & 51.0 & \\
\hline & $5_{2,4}$ & $4_{2,3}$ & 354.899 & -2.9664 & 98.4 & \\
\hline & $5_{4,2}$ & $4_{4,1}$ & 355.029 & -3.3339 & 240.1 & \\
\hline & $5_{4,1}$ & $4_{4,0}$ & 355.029 & -3.3339 & 240.1 & \\
\hline & $12_{1,11}$ & $12_{1,12}$ & 355.042 & -4.7277 & 285.4 & a \\
\hline & $5_{3,3}$ & $4_{3,2}$ & 355.191 & -3.0835 & 157.5 & \\
\hline \multirow[t]{2}{*}{$* *$} & $5_{3,2}$ & $4_{3,1}$ & 355.203 & -3.0834 & 157.5 & \\
\hline & $5_{2,3}$ & $4_{2,2}$ & 356.176 & -2.9618 & 98.5 & \\
\hline \multirow[t]{6}{*}{ HDCO } & $5_{1,4}$ & $4_{1,3}$ & 335.097 & -2.9841 & 56.2 & \\
\hline & $17_{2,15}$ & $17_{2,16}$ & 340.801 & -4.5839 & 517.6 & घ \\
\hline & $3_{1,3}$ & 2,2 & 343.284 & -5.2876 & 25.8 & \\
\hline & $11_{1,10}$ & $11_{1,11}$ & 346.739 & -4.6725 & 219.4 & \\
\hline & $4_{2,2}$ & $5_{0,5}$ & 347.286 & -6.1800 & 62.9 & - \\
\hline & $10_{1,9}$ & $10_{0,10}$ & 355.075 & -5.2163 & 184.3 & - \\
\hline $\mathrm{HDC}^{18} \mathrm{O}$ & $6_{1,6}$ & $5_{1,5}$ & 353.098 & -2.9039 & 67.4 & - \\
\hline \multirow[t]{11}{*}{$\mathrm{D}_{2} \mathrm{CO}$} & $6_{1,6}$ & $5_{1,5}$ & 330.674 & -2.9892 & 61.1 & \\
\hline & $6_{0,6}$ & $5_{0,5}$ & 342.522 & -2.9331 & 58.1 & \\
\hline & $15_{2,13}$ & $15_{2,14}$ & 345.075 & -4.4700 & 370.5 & - \\
\hline & $6_{2,5}$ & $5_{2,4}$ & 349.631 & -2.9553 & 80.4 & \\
\hline & $6_{5,2}$ & $5_{5,1}$ & 351.196 & -3.4129 & 193.7 & - \\
\hline & $6_{5,1}$ & $5_{5,0}$ & 351.196 & -3.4129 & 193.7 & - \\
\hline & $6_{4,3}$ & $5_{4,2}$ & 351.487 & -3.1522 & 145.2 & \\
\hline & $6_{4,2}$ & $5_{4,1}$ & 351.492 & -3.1522 & 145.2 & \\
\hline & $6_{3,4}$ & $5_{3,3}$ & 351.894 & -3.0204 & 107.6 & \\
\hline & $6_{3,3}$ & $5_{3,2}$ & 352.244 & -3.0192 & 107.6 & \\
\hline & $6_{2,4}$ & $5_{2,3}$ & 357.871 & -2.9248 & 81.2 & \\
\hline \multirow[t]{8}{*}{$\mathrm{D}_{2}{ }^{13} \mathrm{CO}$} & $6_{0,6}$ & $5_{0,5}$ & 337.552 & -2.9461 & 57.2 & \\
\hline & $6_{2,5}$ & $5_{2,4}$ & 344.225 & -2.9696 & 79.6 & - \\
\hline & $6_{4,3}$ & $5_{4,2}$ & 345.957 & -3.1670 & 144.7 & घ \\
\hline & $6_{4,2}$ & $5_{4,1}$ & 345.961 & -3.1670 & 144.7 & - \\
\hline & $6_{3,4}$ & $5_{3,3}$ & 346.346 & -3.0352 & 106.9 & \\
\hline & $6_{3,3}$ & $5_{3,2}$ & 346.662 & -3.0340 & 106.9 & \\
\hline & $6_{2,4}$ & $5_{2,3}$ & 351.961 & -2.9406 & 80.3 & - \\
\hline & $6_{1,5}$ & $5_{1,4}$ & 360.989 & -2.8691 & 66.2 & घ \\
\hline
\end{tabular}

Notes. The stars in the left-most column indicates the lines that are blended with each other. The square in the right-most column identifies the lines used in constraining the column density (and for HDCO also the temperature). 
Table A.2. Lines not detected but used to constrain the column densities.

\begin{tabular}{|c|c|c|c|c|c|}
\hline Species & $\begin{array}{c}Q_{1} \\
J_{K_{A} K_{B}}\end{array}$ & $Q_{2}$ & $\begin{array}{c}v \\
\mathrm{GHz}\end{array}$ & $\begin{array}{l}A_{i j} \\
\log _{10}()\end{array}$ & $\begin{array}{r}E_{\mathrm{u}} \\
\mathrm{K} \\
\end{array}$ \\
\hline $\mathrm{H}_{2} \mathrm{CO}$ & $28_{3,25}$ & $28_{3,26}$ & 336.14 & -4.6821 & 1542.1 \\
\hline $\mathrm{H}_{2} \mathrm{C}^{17} \mathrm{O}$ & $188_{3,15}$ & $19_{1,18}$ & 354.222 & -6.0022 & 691.4 \\
\hline & $12_{1,11}$ & $12_{1,12}$ & 355.087 & -4.7276 & 285.4 \\
\hline $\mathrm{H}_{2} \mathrm{C}^{18} \mathrm{O}$ & $12_{1,11}$ & $12_{1,12}$ & 339.498 & -4.7872 & 279.1 \\
\hline $\mathrm{H}_{2}{ }^{13} \mathrm{CO}$ & $29_{3,26}$ & 293,27 & 349.48 & -4.6636 & 1606.4 \\
\hline HDCO & $24_{3,21}$ & $24_{3,22}$ & 348.965 & -4.5321 & 1017.8 \\
\hline $\mathrm{HDC}^{18} \mathrm{O}$ & $2_{2,0}$ & $3_{13}$ & 332.193 & -6.2815 & 41.0 \\
\hline $\mathrm{D}_{2} \mathrm{CO}$ & $7_{3,4}$ & $8_{1,7}$ & 340.380 & -6.1185 & 127.4 \\
\hline
\end{tabular}

\section{Appendix B: Observed spectrum of lines}
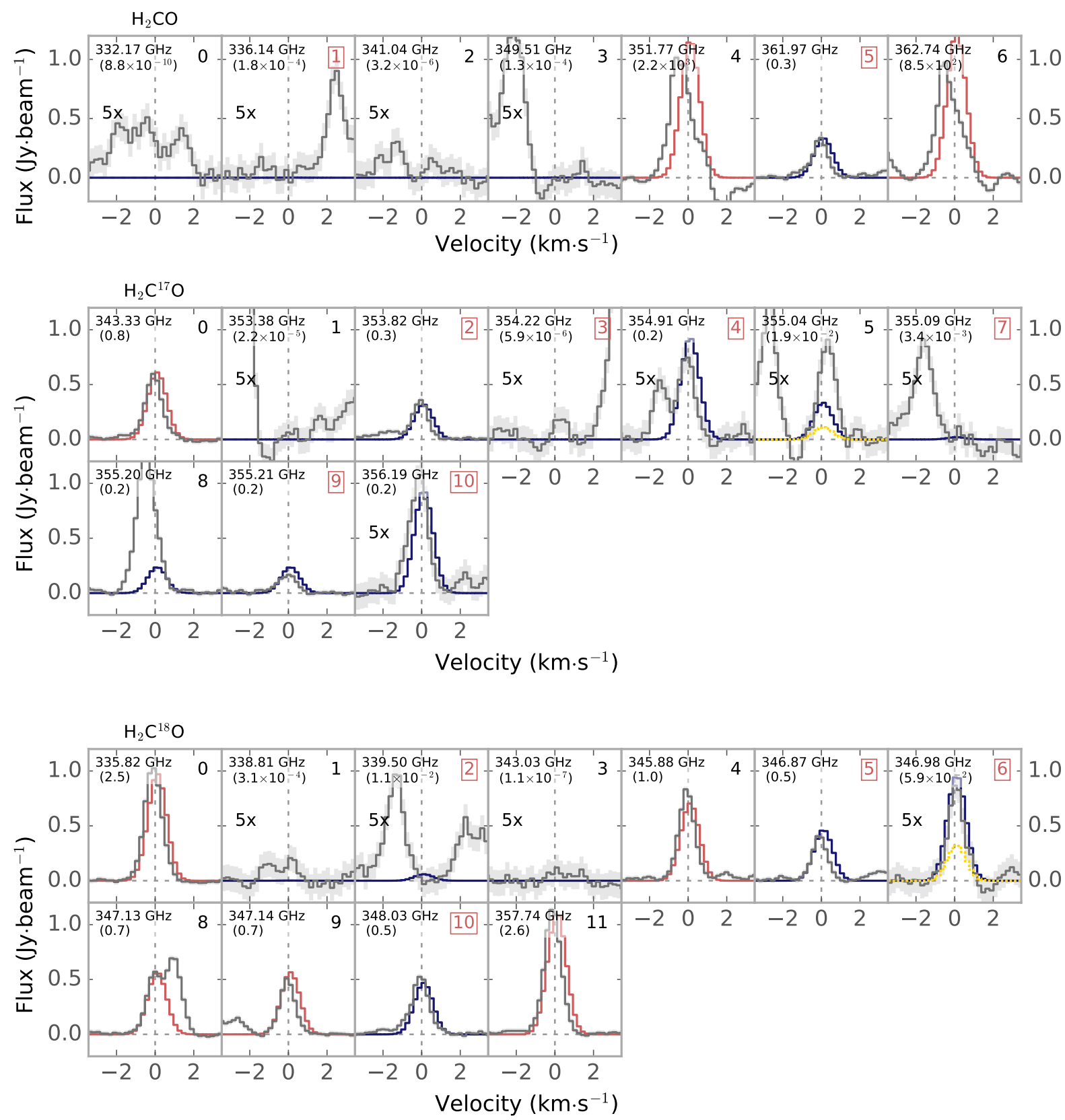

Fig. B.1. All lines of $\left.a) \mathrm{H}_{2} \mathrm{CO}, b\right) \mathrm{H}_{2} \mathrm{C}^{17} \mathrm{O}$, and $\left.c\right) \mathrm{H}_{2} \mathrm{C}^{18} \mathrm{O}$ with the best fit LTE model overplotted in blue. Synthetic spectra in red indicate optical depth beginning to play a significant role i.e., $\tau>0.6$. Numbers in top right corner with red boxes indicate lines used to fit the column density. We note that the two optically thick lines of $\mathrm{H}_{2} \mathrm{CO}$ are the ones with a significant amount of extended emission (covered in van der Wiel, in prep.). The number in parenthesis is the estimated optical depth. Lines in figure $b$ panels 5 and 8 have stronger $\mathrm{H}_{2}{ }^{13} \mathrm{CO}$ lines blended (see panels 9 and 11 in Fig. B.2). 


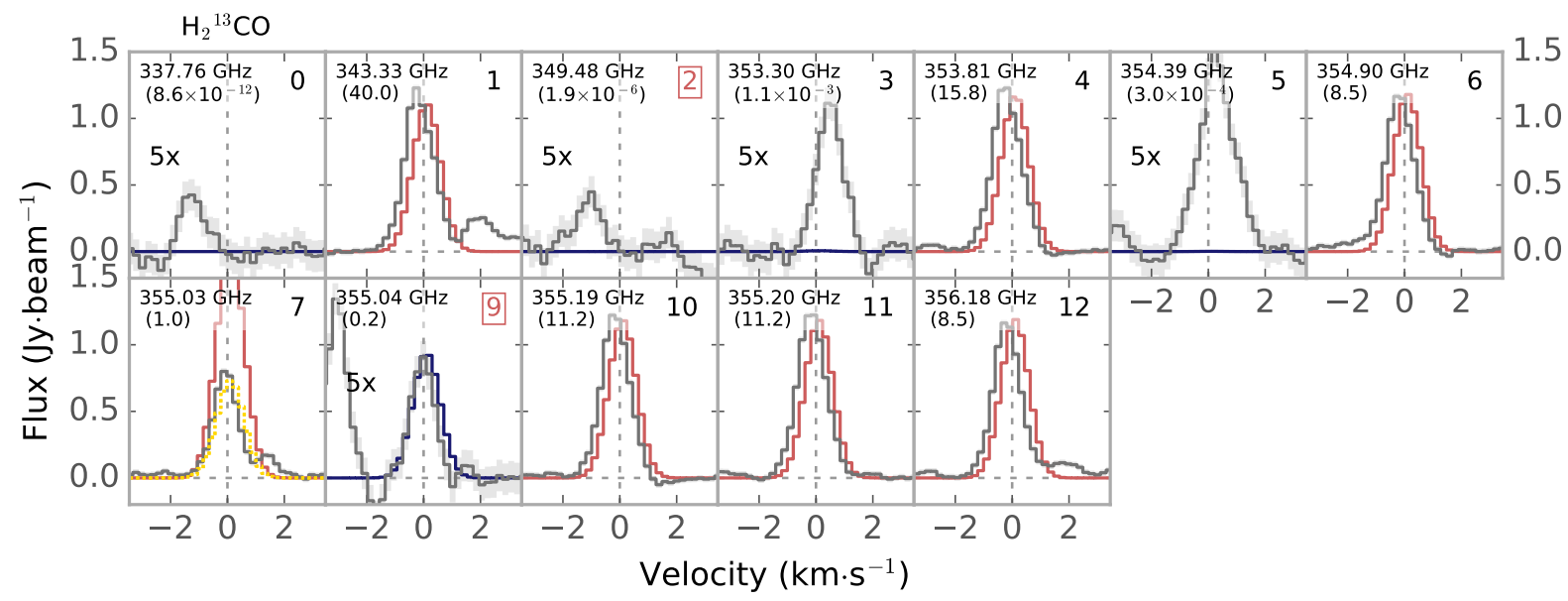

Fig. B.2. All lines of $\mathrm{H}_{2}{ }^{13} \mathrm{CO}$ with the best fit LTE model overplotted. Synthetic spectra in red indicate optical depth beginning to play a significant role i.e., $\tau>0.6$. Numbers in top right corner with red boxes indicate lines used to fit the column density. The number in parenthesis is the estimated optical depth. The line in panels 9 and 11 , at 355.04 and $355.20 \mathrm{GHz}$ could be blended with significantly weaker $\mathrm{H}_{2} \mathrm{C}^{17} \mathrm{O}$ lines $($ see panels 5 and 8 in Fig. B.1).

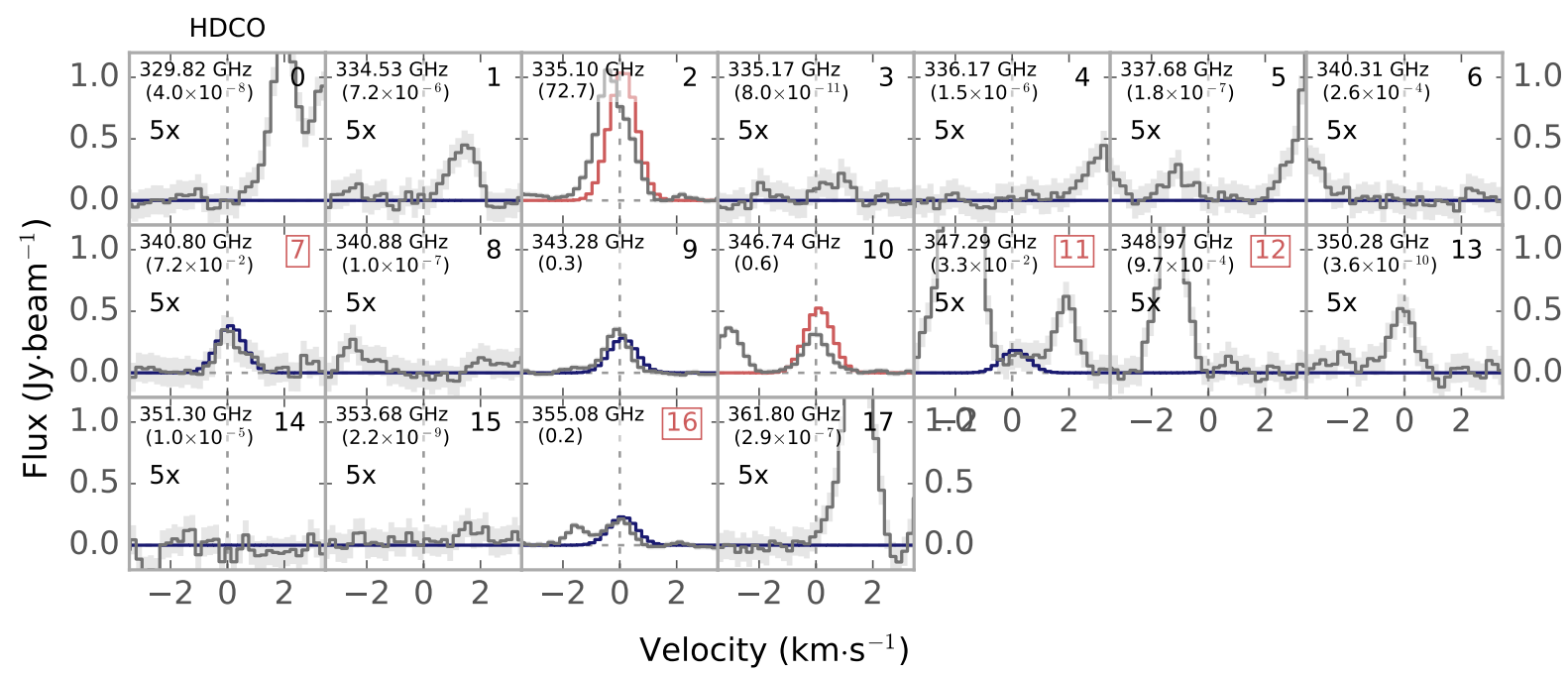

Fig. B.3. All lines of HDCO with the best fit LTE model overplotted. Synthetic spectra in red indicate optical depth beginning to play a significant role i.e., $\tau>0.6$. Numbers in top right corner with red boxes indicate lines used to fit the column density. The number in parenthesis is the estimated optical depth.

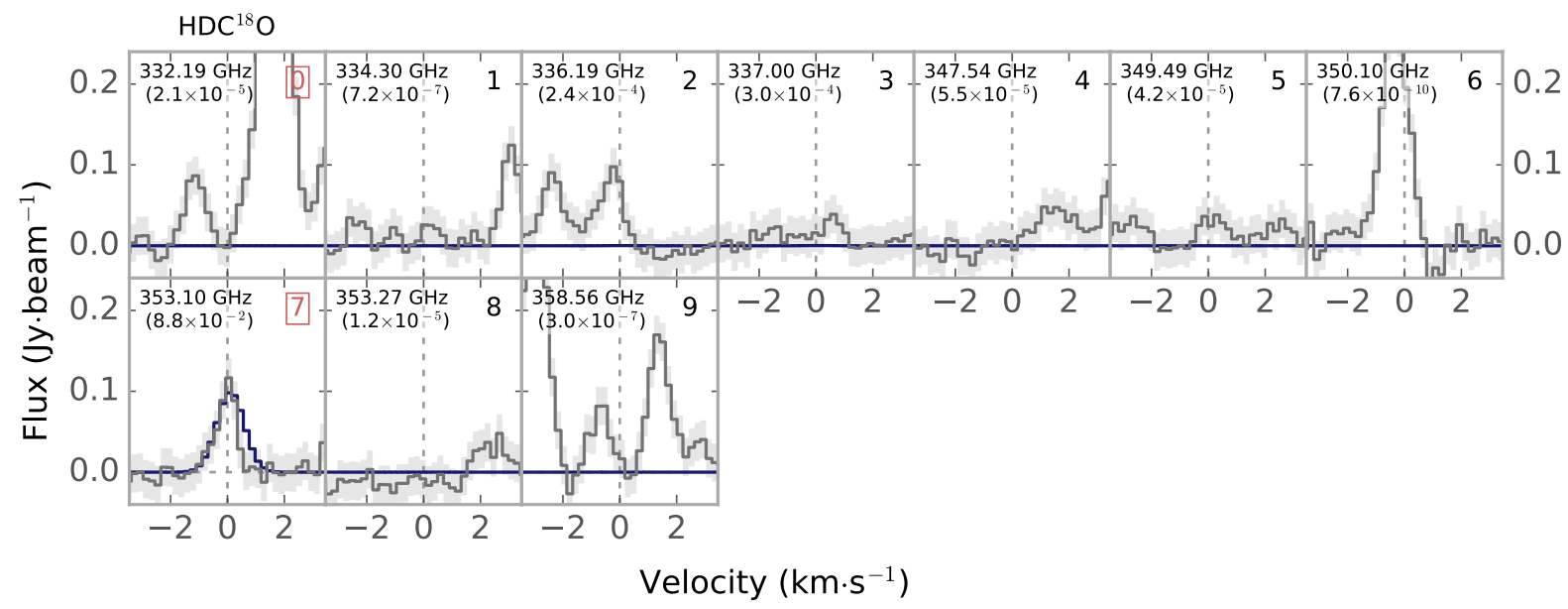

Fig. B.4. All lines of $\mathrm{HDC}^{18} \mathrm{O}$ with the best fit LTE model overplotted. Synthetic spectra in red indicate optical depth beginning to play a significant role i.e., $\tau>0.6$. Numbers in top right corner with red boxes indicate lines used to fit the column density. The number in parenthesis is the estimated optical depth. The emission line at $350.10 \mathrm{GHz}$ is probably methanol. 


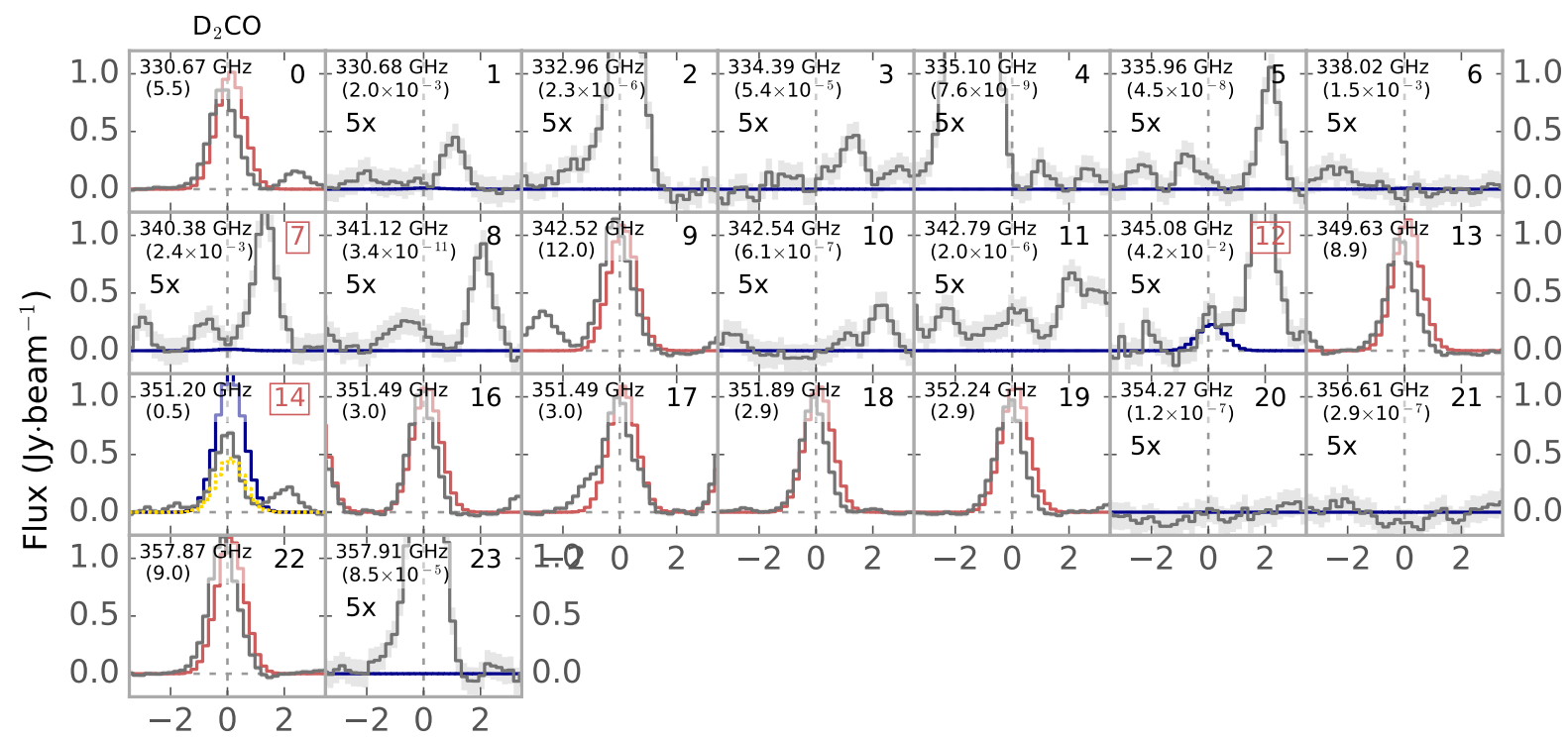

Velocity $\left(\mathrm{km} \cdot \mathrm{s}^{-1}\right)$

Fig. B.5. All lines of $\mathrm{D}_{2} \mathrm{CO}$ with the best fit LTE model overplotted. Synthetic spectra in red indicate optical depth beginning to play a significant role i.e., $\tau>0.6$. Numbers in top right corner with red boxes indicate lines used to fit the column density. The emission line at $332.96 \mathrm{GHz}$ is possibly glycolaldehyde and ethylene glycol, and at $357.91 \mathrm{GHz}$ methyl formate.

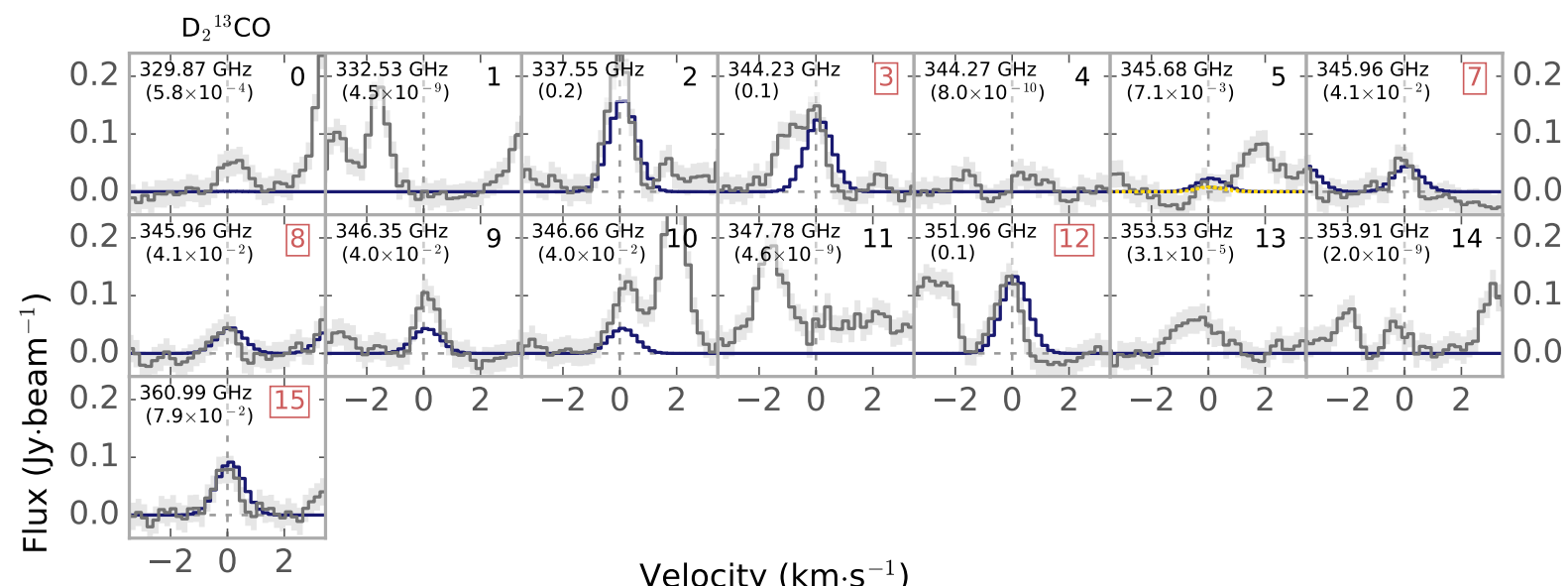

Fig. B.6. All lines of $\mathrm{D}_{2}{ }^{13} \mathrm{CO}$ with the best fit LTE model overplotted. Synthetic spectra in red indicate optical depth beginning to play a significant role i.e., $\tau>0.6$. Numbers in top right corner with red boxes indicate lines used to fit the column density. The number in parenthesis is the estimated optical depth. 